\title{
Damage Simulation of a Random Aggregate Model Induced by Microwave under Different Discontinuous Ratios and Exposure Times
}

\author{
Yang Tang, ${ }^{1}$ Guobin Xu, ${ }^{1}$ Chunlai Qu, ${ }^{2}$ Liying Sun, ${ }^{3}$ and Yu Duan ${ }^{2}$ \\ ${ }^{1}$ State Key Laboratory of Hydraulic Engineering Simulation and Safety, Tianjin University, Tianjin 300072, China \\ ${ }^{2}$ School of Hydroelectricity, Hebei University of Engineering, Handan 056038, China \\ ${ }^{3}$ Applied Mechanics Department, Agricultural University, Beijing 100083, China \\ Correspondence should be addressed to Chunlai Qu; qcl12330@outlook.com
}

Received 9 March 2016; Revised 2 June 2016; Accepted 21 June 2016

Academic Editor: Robert Cerný

Copyright (C) 2016 Yang Tang et al. This is an open access article distributed under the Creative Commons Attribution License, which permits unrestricted use, distribution, and reproduction in any medium, provided the original work is properly cited.

\begin{abstract}
A random aggregate algorithmic method and a numerical model for two-phase materials (composed of quartz and plagioclase) with different discontinuous ratios and irradiation times were studied based on the discrete element method using two-dimensional particle flow code $\left(\mathrm{PFC}^{2 \mathrm{D}}\right)$. The results showed that this algorithm can simulate random irregular aggregate shapes. Furthermore, crack initiation and development and the coalescence process of microwave-induced material damage could be predicted using the discrete element method. After analysis of this study, the micro crack originated from the boundary of the high-absorption-phase plagioclase crystal and expanded around the plagioclase, extending into the quartz material. The crack morphology presented a radial network.
\end{abstract}

\section{Introduction}

Many underground construction developments have been built in recent years due to economic development and the full utilization of water, natural gas, and mineral resources. Blasting and mechanical rock fragmentation methods are commonly used for such construction. The blasting method can cause hidden problems with tunnel stability because of the serious effect on the host rock. The mechanical rock fragmentation method is superior to the blasting method, but there are still some limitations, such as the high cost of the machines, the serious abrasion of cutting tools, and the poor flexibility of rocks. During the entire comminution process, only $1 \%$ efficiency has been observed in terms of the energy utilized to generate new surfaces, resulting in significant energy losses in the comminution process. Microwave treatment has been identified as another potential method for inducing intergranular failures in rock samples [1]. Microwave equipment has considerable advantages, such as lower energy requirements, extremely rapid heating rates, no negative dispersion into the environment, good durability, and a high degree of reliability. The main advantage of microwave technology is that the high-absorbing minerals are affected by the applied energy with minimal wasted energy. Examples of strong microwave absorbers include magnetite, chalcopyrite, and water, and examples of poor microwave absorbers include feldspar, quartz, marble, and ice. The metallic constituents in a rock reflect microwaves into the nonmetallic constituents. Numerous studies of microwaves have been conducted, and an experimental study proved that, in microwave irradiation crushing, rocks will be more crushed when the microwave-irradiated rocks have more power [2]. Olubambi et al. [3] analysed the reason for the intensity change in the rocks and found that when microwaves penetrate rocks, some of the microwave energy that is absorbed by the minerals is then changed to heat. From the microwave irradiation impact strength test and analysis of the rocks, a new finding revealed that the form of continuous microwave irradiation can make the change in rock 
strength more notable [4]. Wang and Forssberg [5] found that particle size has a significant effect on the microwave heating process. Researchers have gained significant knowledge and experience regarding the ability of microwaves to thermally fracture materials. The current status of this research work is still continuing to explore [6]. The entire thermal cracking process is difficult to quantify solely by laboratory experiments; because most minerals are brittle materials with a complex three-dimensional structure, experimental approaches cannot easily yield precise insight into the entire thermal fracture process of ores. In addition, the temperature changes that are produced by microwave irradiation will affect the rock mineral composition and internal microstructure. On a mesoscopic scale, such irradiation led to the formation and development of various internal micro defects; these defects affect the basic mechanical properties of minerals. However, it is difficult to observe the development of the micro cracks in the ore under the influence of temperature and stress.

In this situation, numerical investigations are extremely useful. Previous numerical simulation work using continuum analysis indicated the stress patterns that were generated inside microwaves that were used to treat ore particles $[1$, $7,8]$. However, fractures cannot be explicitly represented using the continuum approach. Compared with the finite element method, the discrete element method has numerous advantages for crack formation; particle flow code (PFC) provided an embodiment of the discrete element method (DEM) for the analysis of rock-mechanics problems and was also a platform for thermal-mechanical analysis. Wanne and Young [9] used $\mathrm{PFC}^{2 \mathrm{D}}$ to study thermally fractured granite and found that the test results and mathematical model results corresponded well. However, the simulation process considered only the temperature change without microwave damage. Ali and Bradshaw [10] used a bonded-particle model in PFC software to predict the effect of microwave power delivery and found that PFC can be used to consider a thermally induced fracture. The above numerical simulation process typically adopted a circular ball with continuous wave application. Based on the static triaxial test, $\mathrm{Wu}$ [11] used the nuclear magnetic resonance method to describe the testing process in detail. Additionally, he combined the theoretical analysis of the aggregate mechanical properties and stated that round particles could not be simulated. Overall, few researchers have conducted damage simulations of a random aggregate model induced by microwaves under different discontinuous ratios and irradiation times. Thus, in this paper, a new random aggregate generation algorithm is developed to simulate a random aggregate shape. The effect of microwave-induced rock damage and the damage mechanism based on the $\mathrm{PFC}^{2 \mathrm{D}}$ numerical software for different discontinuous ratios in a two-component (quartz and plagioclase) rock were studied via numerical simulations. The results provide a new perspective and new method for discontinuous microwave and microwave-induced damage fracture numerical simulation under different damage effects for underground engineering construction.

\section{Random Aggregate Algorithmic Method}

The PFC software used in this paper is $\mathrm{PFC}^{2 \mathrm{D}}$ Version 5.0 [13], whose basic cell is a circle in $\mathrm{PFC}^{2 \mathrm{D}}$. The research results indicate the existing granular materials [14]. Particle shape is an important factor that influences the interaction between particles, as a more irregular particle has a smaller overall stiffness and is more sensitive to the stress state. It is unreasonable to use a circle to simulate a rock specimen. A novel algorithm was used to generate irregular clumps to better characterize the stress variation characteristics of granular materials.

(1) Generate a certain grade of particles in the model boundary (Figure 1(a)). Then, according to (1), randomly generate the centre range of the circle (Figure 1(b)):

$$
\begin{aligned}
& x=x_{b p}+0.0003 * k \\
& y=y_{b p}+0.0003 * k,
\end{aligned}
$$

where $x$ is the centre range of the circle along the $x$ direction, $y$ is the centre range of the circle along with the $y$ direction, $x_{b p}$ is the $x$ coordinate position of an arbitrary particle, $y_{b p}$ is the $y$ coordinate position of an arbitrary particle, and $k$ is a random number, with $k \in(0,1)$.

(2) When the centre range of the circle is confirmed, generate graphics according to (2); when $n$ is equal to 1 , the graphic is an ellipse. When $n$ is greater than or equal to 2 and the value of $n$ increases, the graphics tend to be rectangular. In this paper, $n=1$ is used to generate an irregular block using an elliptic equation algorithm.

Generate an ellipse $E_{i}(i=1)$ using elliptic equation (3). Here, $a$ and $b$ are randomly defined based on (4). The standard oval equation is as follows:

$$
\begin{aligned}
&\left(\frac{x}{a}\right)^{2 n}+\left(\frac{y}{b}\right)^{2 n}=1 \\
&\left(\frac{x}{a}\right)^{2}+\left(\frac{y}{b}\right)^{2}=1 \\
& a=v_{R_{\min }}+v_{R_{a}} * k \\
& b=v_{R_{\min }}+v_{R_{b}} * k \\
& v_{R_{\min }}>0,
\end{aligned}
$$

where $a$ is the long axis, $b$ is the short axis, $v_{R_{\min }}$ is the minimum value of the radius for the particle, $v_{R_{a}}$ and $v_{R_{b}}$ are the maximum value of the radius for the particle, and $k$ is a random number, with $k \in(0,1)$.

(3) If the generated particle is inside the $E_{i}(i=1)$ ellipse boundary, they can be combined as a clump. The number of particles in the clump is generated as $C N_{1}$ (Figure 1(c)); when the first ellipse is generated, the elliptic 


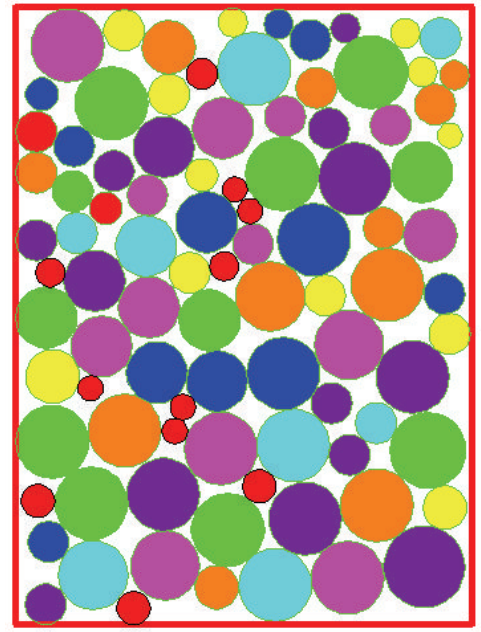

(a) Particle cell

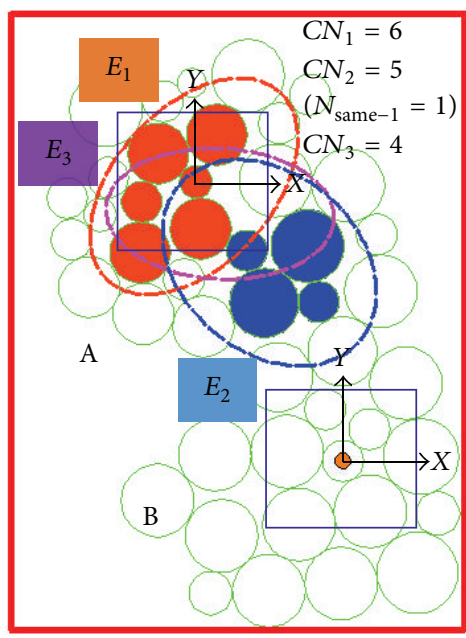

(d) Generate the first clump

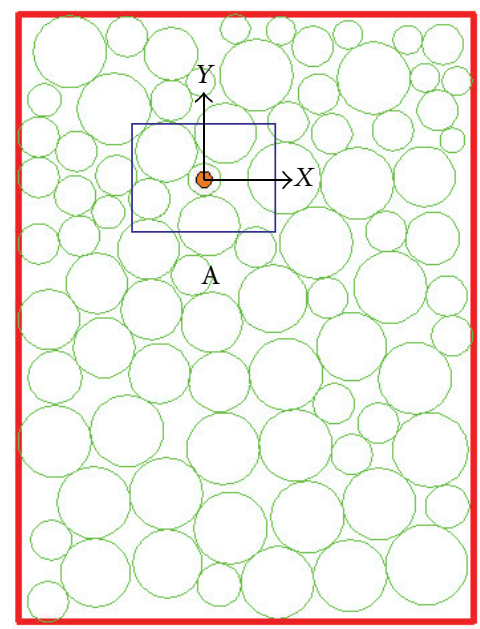

(b) Centre range of the circle

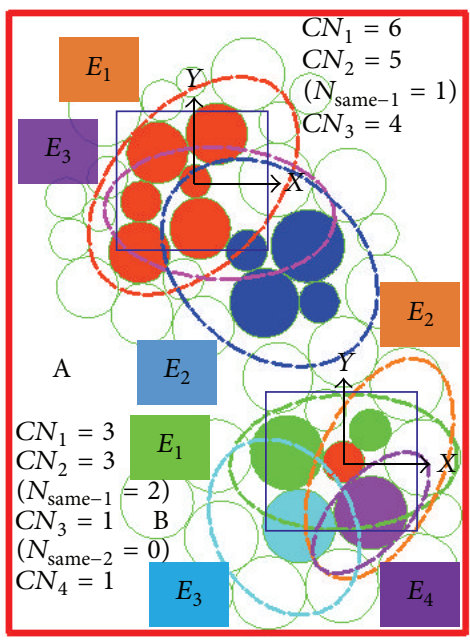

(e) Generate the second clump

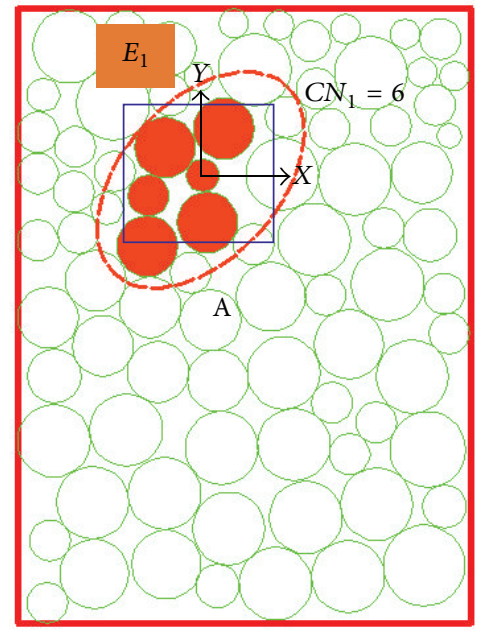

(c) First ellipse

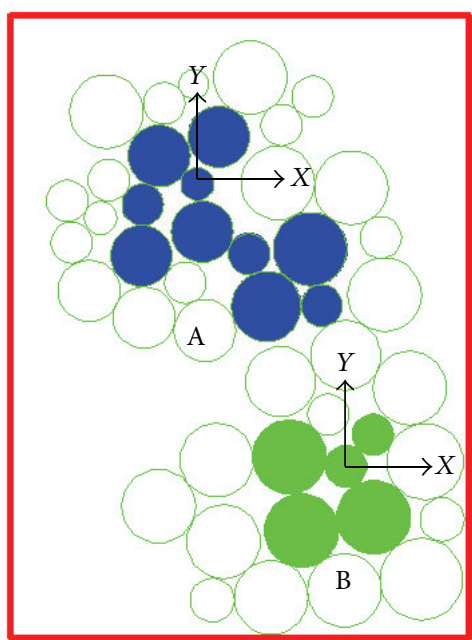

(f) Final shape of the clump

FIGURE 1: Random aggregate algorithm process.

rotating transformation is implemented according to the following equation. Its rotation angle is $\theta$ :

$$
\begin{aligned}
& x=x^{\prime} \cos \theta-y^{\prime} \sin \theta \\
& y=x^{\prime} \sin \theta+y^{\prime} \cos \theta \\
& \theta=\pi * k,
\end{aligned}
$$

where $x^{\prime}$ is the horizontal coordinate value of the first ellipse, $y^{\prime}$ is the longitudinal coordinate value of the first ellipse, $\theta$ is a random rotation angle, and $k$ is a random number, with $k \in(0,1)$.

(4) Make (5) into standard equation (3); specifically, (6) is obtained after rotation. Generate a new ellipse $E_{i}(i \geq 2)$ from (6) and (4); the number of particles in a new ellipse is
$C N_{i}(i \geq 2)$ according to (4); the long axis $a$ and short axis $b$ are random, so the generated ellipse was also random:

$$
\frac{\left(x^{\prime} \cos \theta-y^{\prime} \sin \theta\right)^{2}}{a^{2}}+\frac{\left(x^{\prime} \sin \theta+y^{\prime} \cos \theta\right)^{2}}{b^{2}}=1 \text {. }
$$

(5) According to (7) and (8), the overlapped areas of the ellipse $E_{i}(i \geq 2)$ and the ellipse $E_{i-1}(i \geq 2)$ are defined as $S(n)$; if the number of particles between the new ellipse $E_{i}(i \geq$ 2) and ellipse $E_{i-1}(i \geq 2)$ is identical, then the number of particles is $N(p)_{\text {same }}$. If the same amount of particles is present when $S(n) \neq \emptyset$, then $N(p)_{\text {same }}=0$ :

$$
S(n)=S\left(E_{i}\right) \cap S\left(E_{i-1}\right) \quad(i \geq 2)
$$

$$
N_{\text {same }-j}(j \geq 1)= \begin{cases}0 & S(n)=\emptyset \\ N(p)_{\text {same }} & S(n) \neq \emptyset,\end{cases}
$$




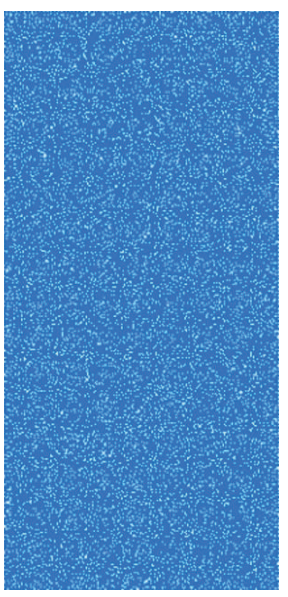

(a) Ball

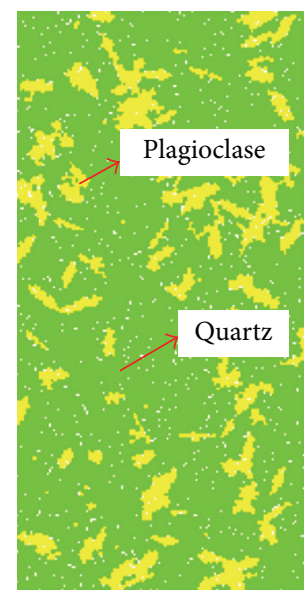

(b) Numerical specimen

Figure 2: Numerical model (typical texture showing microwave absorbent phase (yellow) in a transparent matrix (green) for numerical specimen).

where $S\left(E_{i}\right)$ is the area of ellipse $E_{i}(i \geq 2)$ and $S\left(E_{i-1}\right)$ is the area of ellipse $E_{i-1}(i \geq 2)$.

(6) According to (8), if the new ellipse $E_{i}(i \geq 2)$ and ellipse $E_{i-1}(i \geq 2)$ occur in the overlapped part, then the particle number of the overlapped part is defined as $N_{\text {same-j }}(j \geq 1)$. Based on the criterion combined with (9), the number of particles without overlapping parts in the second ellipse $E_{2}$ is defined as $\Delta N_{1}$. Then, the new clump particle number $C N_{\text {newl }}$ is constituted. Specifically, the new clump particle number $C N_{\text {newl }}$ was equal to the first ellipse particle numbers $C N_{1}$ and $\Delta N_{1}$. Irregular particles in the current stage consist of the new clump particle number $C N_{\text {newl }}$.

Subsequently, according to (6), cyclic rotation in turn generates an ellipse and is combined with (10) until the final number of particles that are generated satisfy (11). Then, the entire cycle will be stopped, and at that moment, all particles in ellipse $E_{i}(i \geq 3)$ are fully contained in ellipse $E_{i-1}(i \geq 3)$. When the irregular clump is generated, it will continue to be the next generation of irregular clumps based on the above method. The volume percentage of irregular particles will be controlled by programming. The process of the random aggregate algorithm is illustrated in Figure 1:

$$
\begin{aligned}
\Delta N_{1} & =C N_{2}-N_{\text {same-1 }} \\
C N_{\text {newl }} & =C N_{1}+\Delta N_{1} \\
\Delta N_{k} & =C N_{i}-N_{\text {same-j }} \quad i \in[3, n) \quad j \in[2, n) \\
C N_{\text {newi-1 }} & =C N_{\text {newl }}+\sum \Delta N_{k} \quad k \in[2, n) \\
\Delta N_{k} & =0 \quad k \in[2, n) \\
C N_{\text {newi }} & \leq C N_{\text {newi-1 }} \quad i \in[3, n) .
\end{aligned}
$$

\section{Numerical Methods}

3.1. Mechanical Model and Micro Parameter. The discrete element method has two basic contact models: the contact bonding model (BCM) and parallel bonding model (BPM). In the BCM, the link that exists between contact points is small and delivers only the force; for the BPM, the main link exists between a particle or block for the contact; thus, the linking is inheritable and the moment is at the same time [15]. At present, the two BCM and BPM links are used [16]. In the BCM, a contact stiffness effect occurs as long as the contact link between particles is maintained. Regardless of linkage to the fracture, the contact link does not influence the stiffness considerably; thus, the material's macro stiffness will not change considerably. For brittle materials, such as concrete and rock, the BPM will be closer to the physical and mechanical properties of the materials [17]. When the stress links are parallel, the BPM model exceeds the link intensity, and links will be fractured; they can be thought of as tiny cracks at this time. Assuming that there are cracks in the normal links, a tension crack is generated. If there are cracks in the tangential link, a shearing crack is generated. The fracture direction was orthogonal to the centre of the connection between particle fractures. Therefore, this paper adopts a parallel bond model to simulate the rock, which is mainly composed of two types of materials: plagioclase and quartz.

Based on the above algorithm, the numerical specimen was created using the procedures of the developed algorithm using the fish language, and the model size is $40 \mathrm{~mm} \times$ $20 \mathrm{~mm}$ (Figure 2), Meng [18] conducted a deep analysis of the rock with microwave irradiation and found that a sample with a height greater than $40 \mathrm{~mm}$ can be completely penetrated by microwaves. It can also satisfy the wholeness of microwave heating well, and therefore, the chosen model size can meet the requirements and ensure warming consistency. The smallest particle size is $0.1 \mathrm{~mm}$, and the ratio of $R_{\max }$ to $R_{\min }$ is set to 1.66 . The entire model can generate a total number of particles for 12,668 balls. The number of clumps is 117 . The entire model was composed of $26 \%$ microwave absorbing mineral (plagioclase) and 74\% transparent matrix (quartz) by area. 
TABLE 1: Mesoscopic parameters of plagioclase.

\begin{tabular}{lc}
\hline Grains & Cement \\
\hline$\rho_{\text {ball }}=2703 \mathrm{~kg} / \mathrm{m}^{3}$ & $\bar{E}_{c}=86.9 \mathrm{GPa}$ \\
$E_{c}=86.9 \mathrm{GPa}$ & $\bar{\sigma}_{c}=\bar{\tau}_{c}=30.6 \pm 5.0 \mathrm{MPa}$ \\
$R_{\min }=0.1 e-3 \mathrm{~m}$ & $R_{\max } / R_{\min }=1.66$ \\
$\mu=0.5$ & \\
\hline
\end{tabular}

$\rho_{\text {ball }}$ is the density of the ball $\left(\mathrm{kg} / \mathrm{m}^{3}\right), \mu$ is the friction coefficient, $R_{\max }$ is the maximum radius of the ball $(\mathrm{mm}), R_{\min }$ is the minimum radius of the ball $(\mathrm{mm}), E_{c}$ is Young's modulus, $\bar{E}_{c}$ is Young's modulus of each parallel bond, $\bar{\sigma}_{c}$ is the normal strength, and $\bar{\tau}_{c}$ is the shear strength.

TABLE 2: Mesoscopic parameters of quartz.

\begin{tabular}{lc}
\hline Grains & Cement \\
\hline$\rho_{\text {ball }}=2648 \mathrm{~kg} / \mathrm{m}^{3}$ & $\bar{E}_{c}=53.3 \mathrm{GPa}$ \\
$E_{c}=53.3 \mathrm{GPa}$ & $\bar{\sigma}_{c}=\bar{\tau}_{c}=30.6 \pm 5.0 \mathrm{MPa}$ \\
$R_{\min }=0.1 e-3 \mathrm{~m}$ & $R_{\max } / R_{\min }=1.66$ \\
$\mu=0.5$ & \\
\hline
\end{tabular}

$\rho_{\text {ball }}$ is the density of the ball $\left(\mathrm{kg} / \mathrm{m}^{3}\right), \mu$ is the friction coefficient, $R_{\max }$ is the maximum radius of the ball $(\mathrm{mm}), R_{\min }$ is the minimum radius of the ball $(\mathrm{mm}), E_{c}$ is Young's modulus, $\bar{E}_{c}$ is Young's modulus of each parallel bond, $\bar{\sigma}_{c}$ is the normal strength, and $\bar{\tau}_{c}$ is the shear strength.

During the numerical simulation process, the common calibration method to select mesoscopic parameters is a trialand-error method. We can obtain reasonable mesoscopic parameters according to a comparison between the results of numerical simulation and actual test results using the trialand-error method. Based on an analysis of the numerical simulation of microwave irradiation, Ali and Bradshaw [1] determined that the tensile strength is more important than the compressive strength in the Brazilian splitting test for ore. It is more appropriate to select a physics experiment with the tensile strength index as the numerical simulation for calibrating microstructure parameters. After choosing different cement strength and tensile strength materials to compare the calibration, the final calibration microscopic parameters of rock are shown in Tables 1 and 2; the strength of the mineral particles obeys a normal distribution in this paper. The aim is to ensure the randomness of the strength of the materials such that the actual rock mineral crystal is realistically simulated.

\subsection{Thermal Model}

3.2.1. Heat Flow Process. In PFC, the thermal material is represented as a network of heat reservoirs (associated with each particle) and thermal pipes (associated with the contacts). A thermal pipe is associated with each contact; heat flow occurs via conduction in the active pipes that connect the reservoirs.

A heat source $(Q)$ and thermal resistance $(\eta)$ are associated with each pipe. The heat source $(Q)$ is derived from the temperature of the microwave conversion. Each pipe has a finite length that is equal to the distance of the two connected particles between the centroids. The thermal resistance $(\eta)$ can be obtained based on

$$
\eta=\frac{1}{2 K}\left(\frac{1-n}{\sum_{N_{b}} V^{(b)}}\right) \sum_{N_{p}} l^{(p)}
$$

where $n$ is the porosity within the numerical specimen, $V^{(b)}$ is the volume of the ball $(b), l^{(p)}$ is the length of the thermal pipe $(p)$, and $N_{b}$ is the number of balls $(b) . N_{p}$ is the number of thermal pipes $(p)$.

3.2.2. Governing Equations for Microwave Heating. The heatconduction equation for a continuum is given by

$$
\frac{\partial\left(K_{i j}\left(\partial T / \partial x_{j}\right)\right)}{\partial x_{i}}+q_{v}=\rho C_{P} \frac{\partial T}{\partial t},
$$

where $K_{i j}$ is the thermal conductivity tensor $(\mathrm{W} / \mathrm{mK}), q_{v}$ is the power density $\left(\mathrm{W} / \mathrm{m}^{3}\right), \rho$ is the density of the material $\left(\mathrm{kg} / \mathrm{m}^{3}\right), C_{P}$ is the heat capacity under constant pressure $(\mathrm{J} / \mathrm{kgK})$, and $T$ is the temperature $(\mathrm{K})$.

Objects undergoing microwave irradiation constantly absorb microwave energy. Dielectric material objects can transform microwave energy; specifically, the electric field energy is converted into heat energy. To be able to quantify microwave-absorbed energy in different materials, the microwave power density is obtained as

$$
P_{d}=2 \pi f \varepsilon_{0} \varepsilon_{r}^{\prime \prime} E_{0}^{2},
$$

where $P_{d}$ is the power density $\left(\mathrm{W} / \mathrm{m}^{3}\right), f$ is the frequency of the microwave irradiation $(\mathrm{Hz}), \varepsilon_{0}$ is the permittivity of free space $\left(8.854 \times 10^{-12} \mathrm{~F} / \mathrm{m}\right), \varepsilon_{r}^{\prime \prime}$ is the dielectric loss factor of the mineral, and $E_{0}$ is the electric field intensity resulting from the microwave power $(\mathrm{V} / \mathrm{m})$.

Equations (13) and (14) illustrate that, by heating objects with microwaves, material properties play a decisive role in certain conditions. The value of the dielectric loss factor of the material determines the speed of the temperature rise in the microwave field.

Combining (13) and (14), the heat-conduction equation is given as

$$
\frac{\partial\left(K_{i j}\left(\partial T / \partial x_{j}\right)\right)}{\partial x_{i}}+2 \pi f \varepsilon_{0} \varepsilon_{r}^{\prime \prime} E_{0}^{2}=\rho C_{P} \frac{\partial T}{\partial t} .
$$

Thus, the temperature rise can be obtained by solving (15) for a given power density and irradiation time.

3.2.3. Thermal-Mechanical Coupling. The electric field can be transformed into heat energy using the governing equations for microwave heating. Then, in PFC, by defining different material properties, the conversion of microwave energy can be achieved by setting the power density and irradiation time. When the microwave energy is converted into heat energy, thermal strains are produced in the $\mathrm{PFC}^{2 \mathrm{D}}$ material by accounting for the thermal expansion of the particles. Given 
a temperature change $(\Delta T)$, the change in each particle radius $(R)$ is given by

$$
\Delta R=\alpha R \Delta T,
$$

where $\alpha$ is the coefficient of thermal expansion that is associated with the particle. This paper adopts a parallel bonding model to simulate a rock that is mainly composed of two types of materials: quartz and plagioclase. If a parallel bond is present at the contact associated with a pipe, then we obtain

$$
\Delta \bar{F}^{n}=-\bar{K}^{n} A \Delta U^{n}=-\bar{K}^{n} A(\bar{\alpha} \bar{L} \Delta T),
$$

where $\bar{K}^{n}$ is the normal bond stiffness, $A$ is the area of the bond cross section, $\bar{\alpha}$ is the expansion coefficient, and $\bar{L}$ is the bond length (assumed to be equal to the distance between the centroids of the two particles at the ends of the pipe associated with the bond).

3.2.4. Boundary Conditions. In general, boundary conditions require a description of the local boundary in terms of an outwardly directed normal unit vector. However, in the $\mathrm{PFC}^{2 \mathrm{D}}$ material, there is no explicit representation of the boundary (unlike in a continuum code, where zone surfaces comprise the boundary). This problem is overcome by specifying the power being input to a boundary surface instead of the flux. In this paper, the external boundaries of the thermal model were considered thermally insulated because the microwave irradiation times were quite short; therefore, the convection and radiation were not incorporated into the model.

\subsection{Thermal Property Data and Numerical Simulation Condi-} tions. Ali and Bradshaw [19] demonstrated that the thermal induced stress within a material after microwave treatment depends largely on its thermal properties. Therefore, in PFC, each thermal property was written as a function in fish language and was incorporated into the numerical model.

3.3.1. Power Density and Irradiation Time. According to (15), the power density and irradiation time are two important parameters for realizing the transformation of energy. Numerous publications have explained the influence of the power density $[6,10]$. To avoid repetitive experiments, this paper used the form power densities of $1 \times 10^{9} \mathrm{~W} / \mathrm{m}^{3}$; these irradiation times (between 0.05 and $0.4 \mathrm{~s}$ ) were chosen from previous simulation results [10]. Because the material that we chose is different from the previous simulation, we used microwave irradiation times between 0.2 and $0.8 \mathrm{~s}$ for a power density of $1 \times 10^{9} \mathrm{~W} / \mathrm{m}^{3}$.

3.3.2. Microwave Frequency. According to standard industry microwave frequencies, $2.45 \mathrm{GHz}$ was chosen; a wavelength under a $2.45 \mathrm{GHz}$ microwave frequency can travel through the rock specimen [18].

3.3.3. Thermal Property Data. The specific heat capacity, thermal conductivity, and thermal expansion coefficient of
TABLE 3: Thermal property data for quartz and plagioclase as a function of temperature.

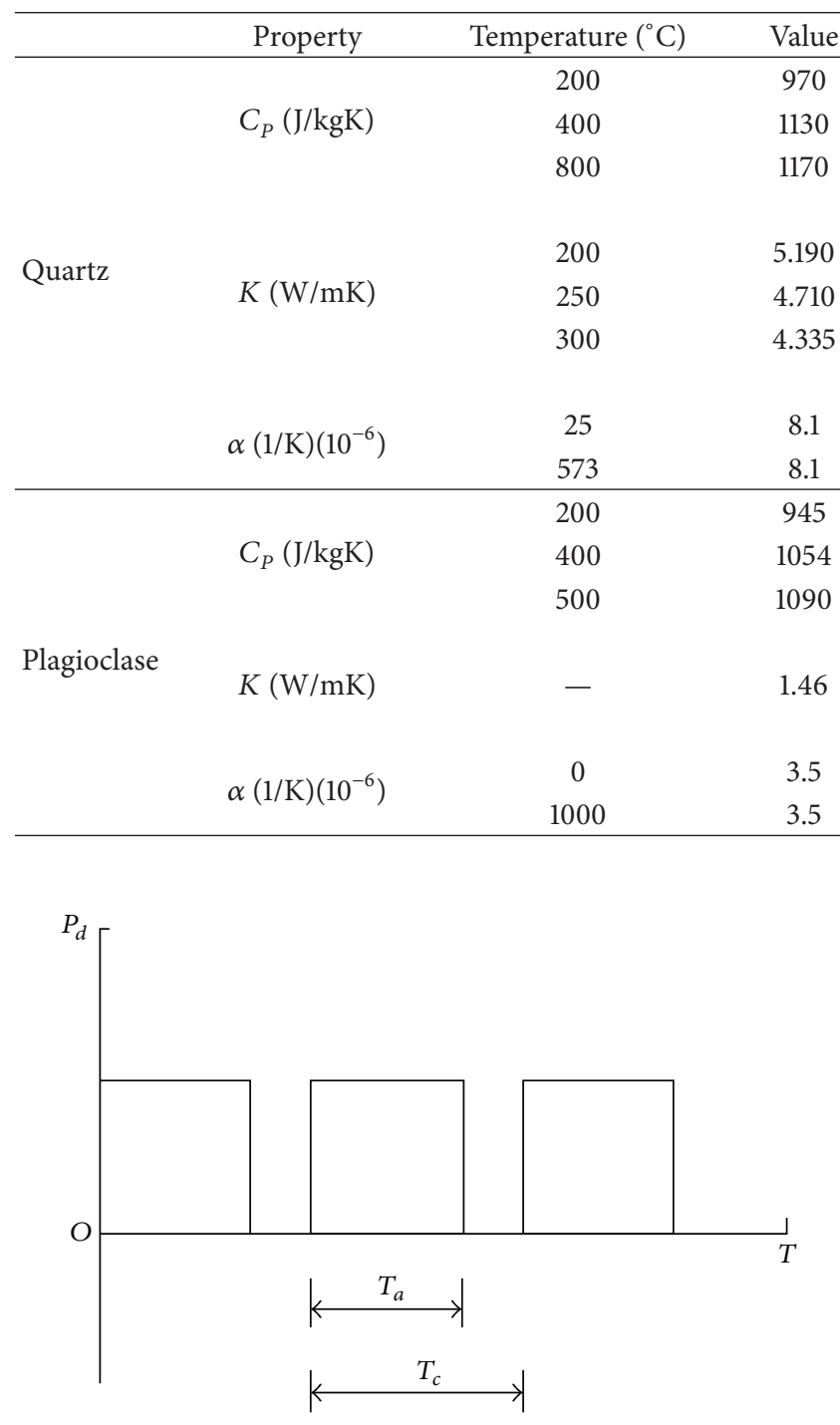

FIgURE 3: Power density change of a square wave over time.

the materials were obtained from Meisels et al. [12] and are tabulated in Table 3.

3.3.4. Numerical Simulation Conditions. In this study, we define the discontinuous ratio $D_{i}$ as

$$
D_{i}=\frac{T_{a}}{T_{c}}
$$

where $T_{a}$ is the time for applying the microwaves and $T_{c}$ is one period. $D_{i}$ expresses the percentage of time applied to the microwave power in one period. The power density change of a square wave over time is illustrated in Figure 3. Here, when $T_{a}$ is equal to $T_{c}$, the microwaves belong to a continuous process. Different discontinuous ratios are shown in Table 4. The irradiation times are equal to $0.2,0.5$, and $0.8 \mathrm{~s}$ in a variety of different discontinuous ratio conditions. 
TABLE 4: Discontinuity ratios in the test.

\begin{tabular}{llllll}
\hline$D_{i}$ & $D_{1}$ & $D_{2}$ & $D_{3}$ & $D_{4}$ & $D_{5}$ \\
\hline $1 \times 10^{9} \mathrm{~W} / \mathrm{m}^{3}$ & 0.2 & 0.4 & 0.6 & 0.8 & 1.0 \\
\hline
\end{tabular}

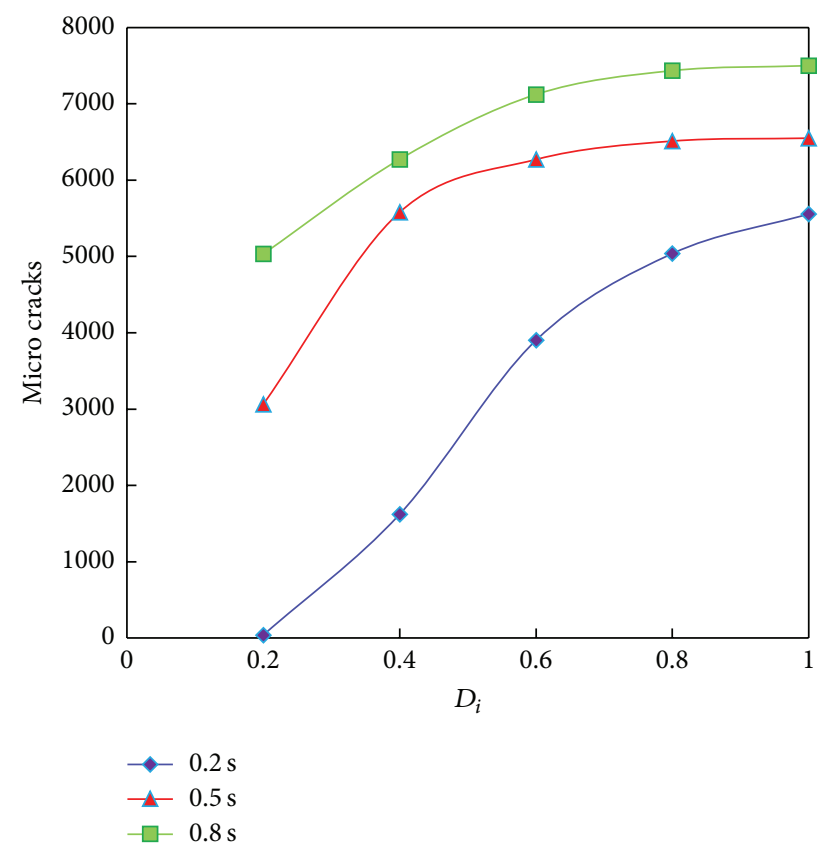

FIGURE 4: Relationship between the number of cracks and the discontinuous ratio under different irradiation times.

3.3.5. Thermal Loading. Given the different thermal properties of the material (quartz and plagioclase) and using the irradiation time, the temperature field varied with the thermal load. Plagioclase was used as a wave absorbent phase, and quartz was used as a transparent phase.

\section{Results and Discussion}

4.1. Effect of Microwave-Induced Damage under Different Discontinuous Ratios. As shown in Figure 4, with the same irradiation time, a different number of cracks were observed for different discontinuous ratios. The number of cracks increased gradually with increases in the discontinuous ratios.

When the irradiation time is equal to $0.2 \mathrm{~s}$, relatively few cracks are generated when the discontinuous ratio is less than 0.2 . The number of cracks increases gradually with an increasing discontinuous ratio, and microwaves belong to the continuous process when the discontinuous ratio is equal to 1. The number of cracks in the continuous wave condition reaches its peak value compared to when the discontinuous ratio is equal to 0.2 operating conditions, when the crack number increased nearly 142 times. When the irradiation time is equal to $0.5 \mathrm{~s}$, the number of cracks is 3,063 when the discontinuous ratio is equal to 0.2 . The number of cracks is increased by 78 times for an irradiation time of $0.5 \mathrm{~s}$ compared to $0.2 \mathrm{~s}$ with the same discontinuous ratio of 0.2 . When the discontinuous ratio is increased to 0.4 with an irradiation time of $0.5 \mathrm{~s}$, the total number of cracks is 5,579 . The effect of microwave-induced damage is approximately equal to that of the condition when the irradiation time is equal to $0.2 \mathrm{~s}$ and the discontinuous ratio is equal to 1 .

Subsequently, when the irradiation time is equal to $0.8 \mathrm{~s}$ and the discontinuous ratio is equal to 0.6 , the effect of microwave-induced damage is 1.09 times that when the irradiation time is equal to $0.5 \mathrm{~s}$ and the discontinuous ratio is equal to 1 . These phenomena indicate that the discontinuity of microwaves has a significant influence on the damage of quartz-plagioclase. Microwave damage is more obvious with an increasing discontinuous ratio. These results indicate that the induced damage for different square waves differs for different discontinuous ratios and different irradiation times, and the effect of the discontinuity of the square wave is considerable. For example, with an irradiation time of $0.5 \mathrm{~s}$ and a discontinuous ratio of 1 , the continuous wave exposure effect can be replaced by an irradiation time of $0.8 \mathrm{~s}$ and a discontinuous ratio of 0.6 .

4.2. Mechanism of Microwave-Induced Damage under Different Discontinuous Ratios. Because of the length of the space and damage morphology, the numerical simulation is highly similar for different discontinuous ratio simulations of quartz-plagioclase treated at a power density of $1 \times 10^{9} \mathrm{~W} / \mathrm{m}^{3}$; Table 5 provides the partial results. Combining Figure 4 with Table 5 illustrates that microwave-induced damage is not identical under different discontinuous ratios; when the discontinuous ratio is more than 0.2 and the irradiation time exceeds $0.5 \mathrm{~s}$, microwave-induced damage is more obvious with increases in the discontinuous ratio and irradiation time.

In addition, under the injury conditions, the tensile crack originated from the outer boundary of the plagioclase; few damage fractures were observed in the plagioclase. Most of the fractures were located in the interface of the plagioclase and quartz and around the absorption phase when the plagioclase expands, extending into the quartz material. Finally, a radial network tensile crack is observed because of the composition and thermal property differences in the plagioclase and quartz materials. The dielectric plagioclase crystal particles will absorb microwave and translated microwave energy into heat, and the deformations of the various particles differ when plagioclase and quartz absorb the microwave energy; the temperature rapidly increases with the action of thermal energy (see Figure 5; this paper lists the temperature distribution under different discontinuity ratios for an irradiation time of $0.8 \mathrm{~s}$ ). Figure 5 illustrates that the temperature of the plagioclase material gradually increases with increases in the discontinuity ratio; the temperature difference is obvious compared to that of quartz materials. Thus, a tensile stress is caused by the microwave energy in the material; with a greater temperature gradient, the plagioclase crystal particles will expand by heat, resulting in a greater tensile stress around the quartz structure. Jones et al. [20] used a continuum method to confirm that the tensile stress exists around the grain boundary. When the tensile stress reached and exceeded the bond strength between the particles, the bond of mineral particles broke along the interface between 
TABLE 5: Failure patterns of microwave-induced damage in plagioclase-quartz for $D_{i}=0.2$ and 0.8 .

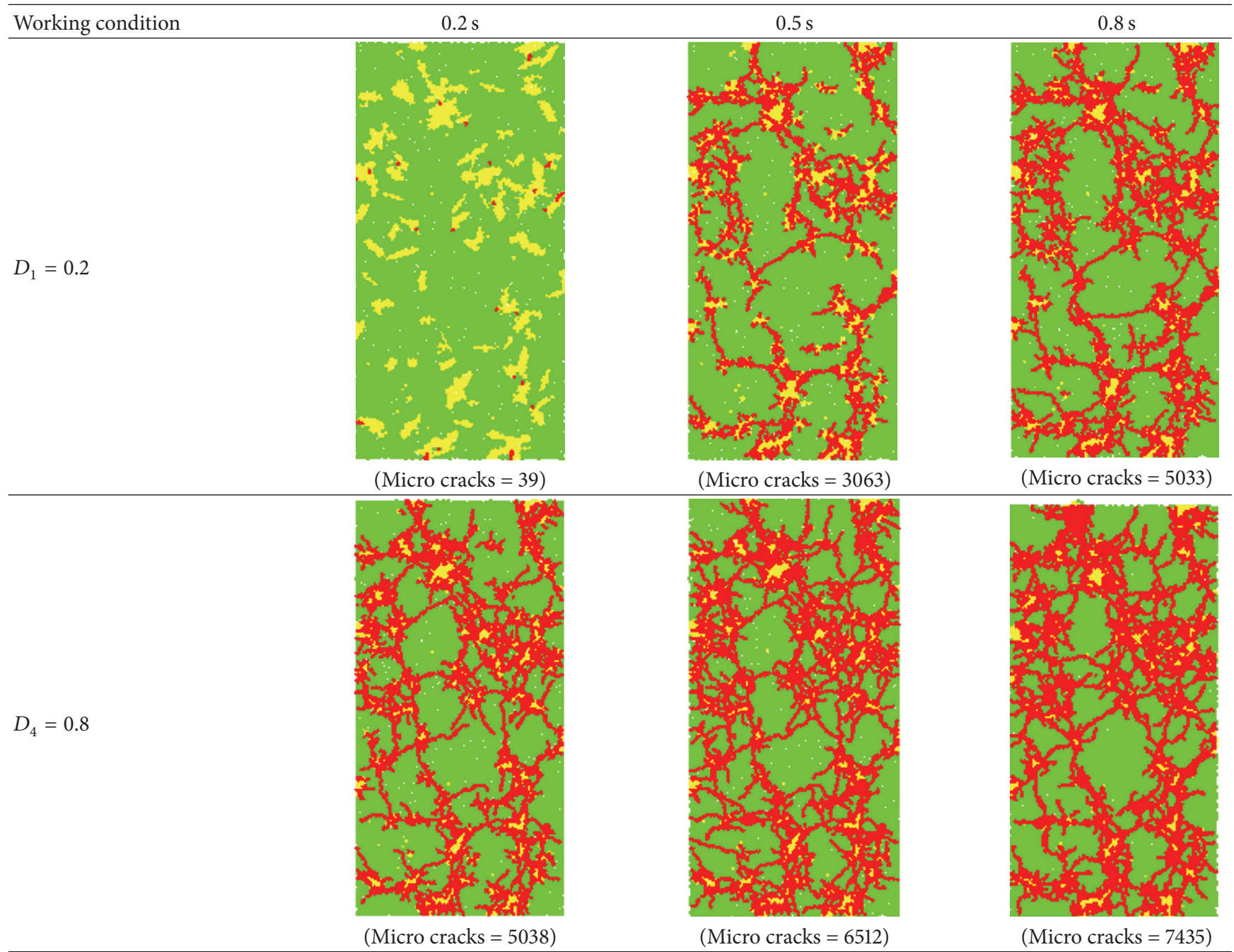

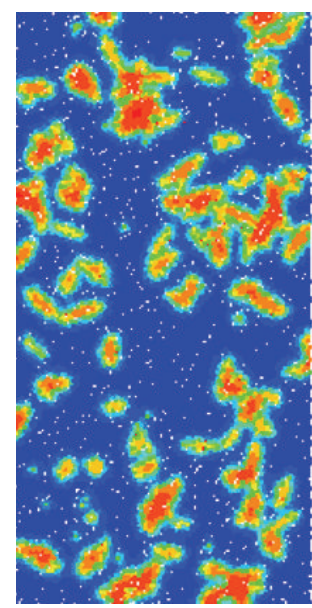

$D_{1}=0.2$

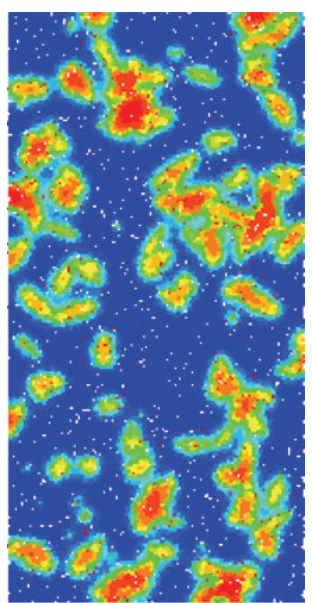

$D_{2}=0.4$

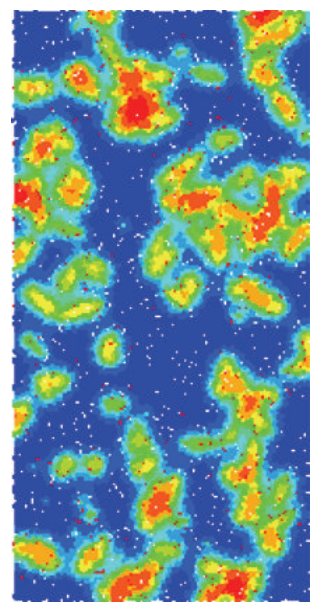

$D_{3}=0.6$

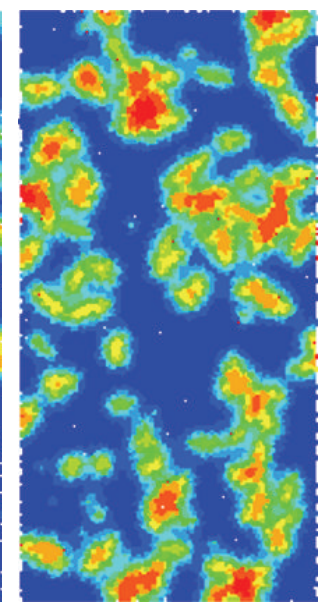

$D_{4}=0.8$

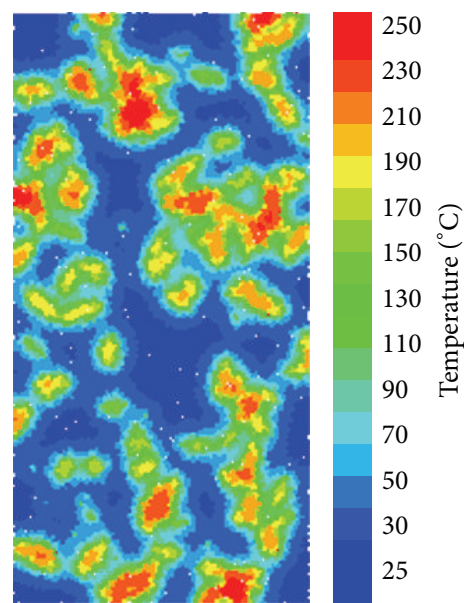

$D_{5}=1.0$

FIgURE 5: Temperature distribution with different discontinuities ratios for an irradiation time of $0.8 \mathrm{~s}$. 
this edge, resulting in micro cracks. After crack formation, the stress in the inner structure will redistribute around the breakage, leading to a micro crack continuously extending to the quartz and continuously expanding along the places with lower bond strength, thus inducing more densely connected crack initiation and development in addition to larger crack formation. Finally, the radiation pattern is presented.

With increasing irradiation time, the effect of microwave damage is poor when the discontinuous ratio is equal to 0.2 and the irradiation time is equal to $0.2 \mathrm{~s}$, and there are no discernable radial cracks. With increases in the discontinuous ratio and irradiation time, cracks gradually developed as in the diffusion network, and the effect of microwave damage is greater. Based on the above analysis, the nature of mass rock damage induced by microwaves results from differences in the discontinuous ratio of the microwaves; different energies were obtained under various conditions.

4.3. Test Verification. To verify the accuracy of the numerical simulation results and because of the destruction of the morphological similarities, this paper selected the numerical simulation condition of $D_{i}=0.8$ as the comparison group (Table 5).

Temperature changes will affect the mineral composition and microstructure of the rock; thus, the formation and development of all types of internal micro defects at the microscopic level are presented. However, it is difficult to observe the development of the micro crack network in rock under the influence of temperature and stress. Thus, the rock specimens were first irradiated by microwaves, and then, the internal damage and micro morphology were observed using CT and SEM technology for the different irradiation times. Finally, the test results were compared with the numerical simulation results under the $D_{i}=0.8$ condition.

4.3.1. Microwave Heating Specimen. The experiment on granite was performed at $2.45 \mathrm{GHz}$; an open-ended square waveguide acted as the applicator. The exposure condition is that the power is equal to $1 \mathrm{~kW}$ and the irradiation time is equal to 0 , 20,50 , and $80 \mathrm{~s}$; microwave power is intermittent with a discontinuous ratio of $D_{i}=0.8$. The rock specimen was placed in the centre of the microwave oven cavity. A microwave heated rock is shown in Figure 6. Real irradiation times equal to 0,20, 50 , and $80 \mathrm{~s}$ correspond to numerical simulation irradiation times of $0,0.2,0.5$, and $0.8 \mathrm{~s}$, respectively.

4.3.2. Verification. CT images of rock under different irradiation times are shown in Figure 7; as the irradiation time increases, the shape of the crack changes continuously, illustrating that the interior of the rock mass undergoes deformation. In addition, cracks are continuously changing, which illustrates that microwave-induced damage is an irreversible process.

From Figure 7 we can find that the experiments show significantly fewer cracks than the simulations, which is mainly due to the fact that the mineralogy used for the modelling is a simplified version of reality. It was preferable to simulate simplified models of the ore particles in order to

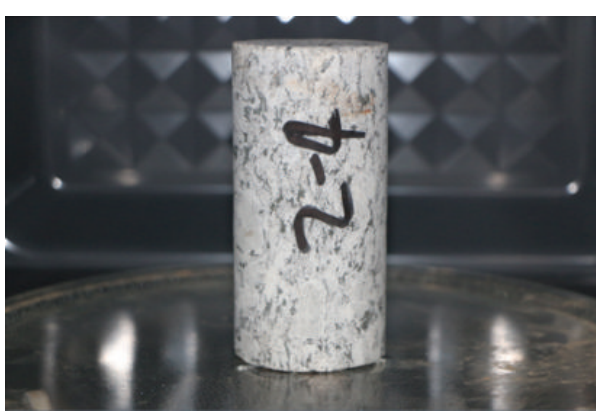

FIgURE 6: Microwave heated rock.

elucidate the general effects of microwave treatment better. In addition, the stochastic distribution model was adopted in the numerical simulation, and it was inevitable to making the difference compared to the results of physical test. There exists another reason that the simulation time was not consistent with the actual time, which leads to the differences of crack numbers between the numerical model and the actual model. But the macro change trend of the cracks can be reflected by the simulation on some level. The rock images finally present a tensile failure form. The failure form of CT images corresponds with the result of the numerical simulation comparison group.

We used scanning electron microscopy (SEM) to better observe the crack growth between mineral particles. The rock micro crack morphology under different microwave irradiation times (Figure 8) illustrates that, with increasing microwave irradiation time, thermal cracking typically occurs on the cement surface of mineral particles. There is a trend to form a variety of mesh structures; the development of this network fracture structure is typically terminated by a section of cement in the region, mainly due to the fact that the expansion anisotropy and thermal mismatch of the irregular plagioclase crystal closed angle and pointedness will develop stress concentration points. Under the thermal stress action, when the local stress exceeds the tensile strength of the rock, a crack in the rock is continuously extended, and the final crack is present in the shape of the scattered distribution.

The test results are consistent with the results of the numerical simulation, demonstrating that the results of the numerical simulation are accurate. Meisels et al. [12] also proved this view (Figure 9).

\section{Conclusions}

The following results were obtained using the discrete element method to simulate microwave-induced damage under different discontinuous ratios and irradiation times:

(1) A new method of random rock clump generation was established based on the discrete element method. This method can accurately simulate irregular shapes.

(2) The microwave-induced damage effect and failure mode under different discontinuous ratios were elucidated. The discontinuous ratio and irradiation time 


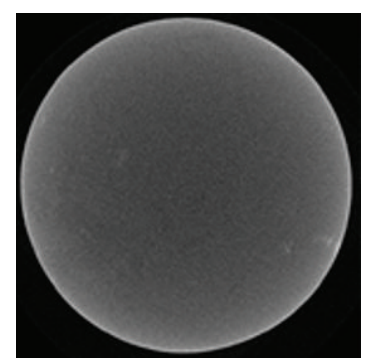

$0 \mathrm{~s}$

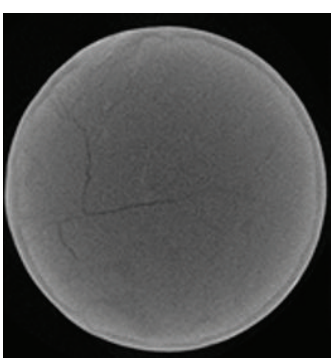

$20 \mathrm{~s}$

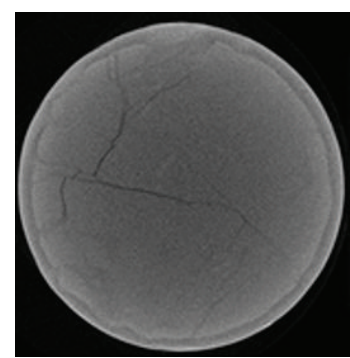

$50 \mathrm{~s}$

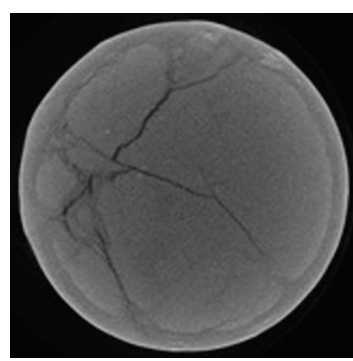

$80 \mathrm{~s}$

FIGURE 7: CT images at different irradiation times.

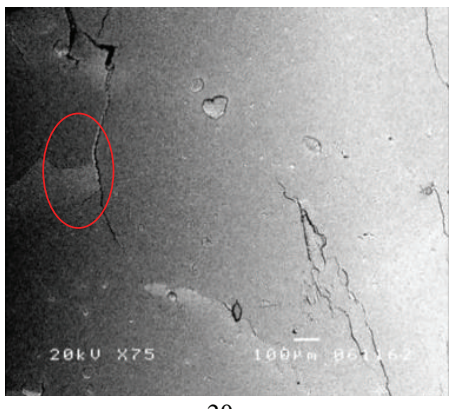

$20 s$

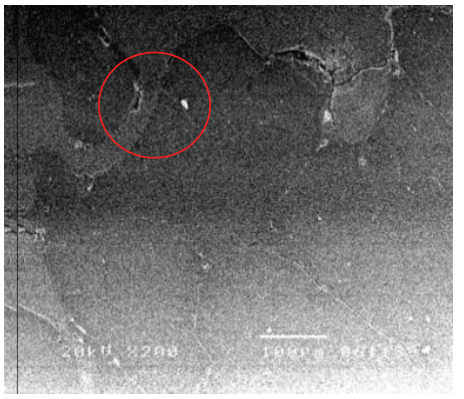

$20 \mathrm{~s}$

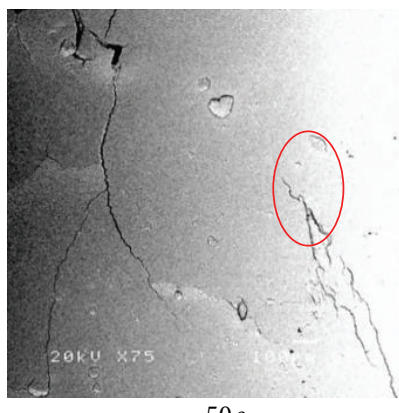

$50 \mathrm{~s}$

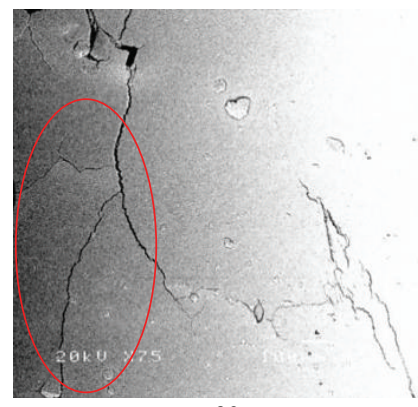

$80 \mathrm{~s}$

(a) Crack development under 75x magnification

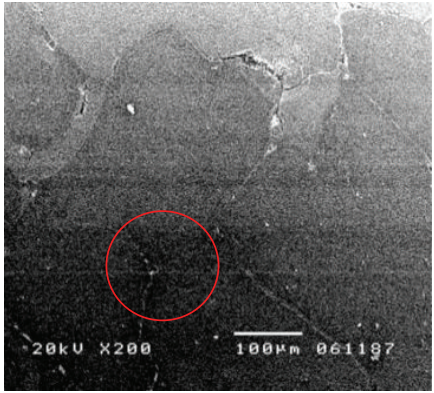

$50 s$

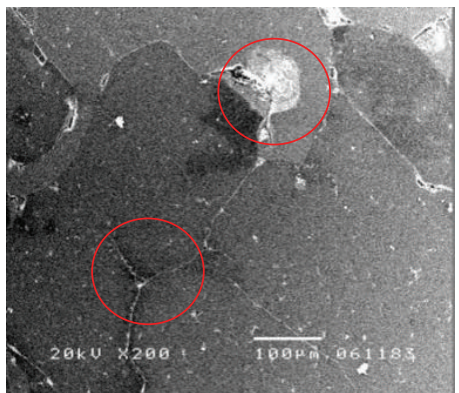

$80 s$

(b) Crack development under 200x magnification

FIGURE 8: Micro cracks under different magnifications of internal rock damage.

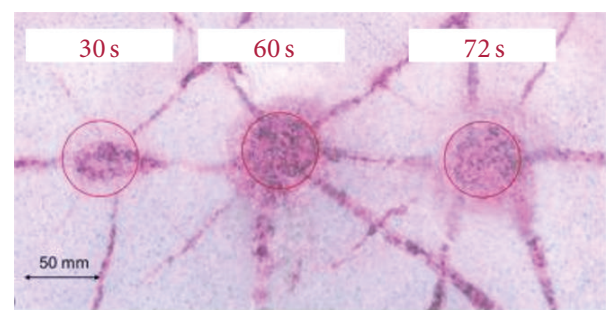

FIGURE 9: Crack propagation for granite at different times [12].

have an important effect on microwave-induced damage. A limited number of micro fractures inside the absorption phase of the plagioclase were observed with increasing discontinuous ratios and irradiation times. Most micro fractures originated from the interface between plagioclase and quartz and around the absorption phase plagioclase, extending into quartz, and finally presenting as a radial network tension crack.

(3) The microwave damage effects differed under different microwave discontinuous ratios; they can be used to mutually transmit based on discontinuous ratios and irradiation times to better utilize resources.

\section{Competing Interests}

The authors declare that there are no competing interests regarding the publication of this paper. 


\section{Acknowledgments}

This research was supported by the Science Fund for Creative Research Groups of the National Natural Science Foundation of China (Grant no. 51321065).

\section{References}

[1] A. Y. Ali and S. M. Bradshaw, "Quantifying damage around grain boundaries in microwave treated ores," Chemical Engineering and Processing: Process Intensification, vol. 48, no. 11-12, pp. 1566-1573, 2009.

[2] I. V. Ebo Sijie, E. I. Ashi, and G. K. Wei Tuote, New Rock Breaking Method, Tao Zaipu; China Industry Press, Beijing, China, 1962 (Chinese).

[3] P. A. Olubambi, J. H. Potgieter, J. Y. Hwang, and S. Ndlovu, "Influence of microwave heating on the processing and dissolution behaviour of low-grade complex sulphide ores," Hydrometallurgy, vol. 89, no. 1-2, pp. 127-135, 2007.

[4] G. Scott, S. M. Bradshaw, and J. J. Eksteen, "The effect of microwave pretreatment on the liberation of a copper carbonatite ore after milling," International Journal of Mineral Processing, vol. 85, no. 4, pp. 121-128, 2008.

[5] Y. Wang and E. Forssberg, "Dry comminution and liberation with microwave assistance," Scandinavian Journal of Metallurgy, vol. 34, no. 1, pp. 57-63, 2005.

[6] G. Wang, P. Radziszewski, and J. Ouellet, "Particle modeling simulation of thermal effects on ore breakage," Computational Materials Science, vol. 43, no. 4, pp. 892-901, 2008.

[7] J. B. Salsman, R. L. Williamson, W. K. Tolley, and D. A. Rice, "Short-pulse microwave treatment of disseminated sulfide ores," Minerals Engineering, vol. 9, no. 1, pp. 43-54, 1996.

[8] D. N. Whittles, S. W. Kingman, and D. J. Reddish, "Application of numerical modelling for prediction of the influence of power density on microwave-assisted breakage," International Journal of Mineral Processing, vol. 68, no. 1-4, pp. 71-91, 2003.

[9] T. S. Wanne and R. P. Young, "Bonded-particle modeling of thermally fractured granite," International Journal of Rock Mechanics and Mining Sciences, vol. 45, no. 5, pp. 789-799, 2008.

[10] A. Y. Ali and S. M. Bradshaw, "Bonded-particle modelling of microwave-induced damage in ore particles," Minerals Engineering, vol. 23, no. 10, pp. 780-790, 2010.

[11] L. P. Wu, Study on the Microfabric of Coarse Aggregate, Yangtze River Scientific Research Institute, Wuhan, China, 2007 (Chinese).

[12] R. Meisels, M. Toifl, P. Hartlieb, F. Kuchar, and T. Antretter, "Microwave propagation and absorption and its thermomechanical consequences in heterogeneous rocks," International Journal of Mineral Processing, vol. 135, pp. 40-51, 2015.

[13] Itasca Consulting Group Inc, PFC2D (Particle Flow Code in 2 Dimensions): Version 5.0. ICG, Itasca Consulting Group Inc, Minneapolis, Minn, USA, 2014.

[14] G.-C. Cho, J. Dodds, and J. C. Santamarina, "Particle shape effects on packing density, stiffness, and strength: natural and crushed sands," Journal of Geotechnical and Geoenvironmental Engineering, vol. 132, no. 5, pp. 591-602, 2006.

[15] Itasca Consulting Group Inc, PFC2D (Particle Flow Code in 2 Dimensions): Version 3.0, Itasca Consulting Group Inc, Minneapolis, Minn, USA, 2002.

[16] J. F. Hazzard, R. P. Young, and S. C. Maxwell, "Micromechanical modeling of cracking and failure in brittle rocks," Journal of
Geophysical Research: Solid Earth, vol. 105, no. 7, Article ID 2000JB900085, pp. 16683-16697, 2000.

[17] N. Cho, C. D. Martin, and D. C. Sego, "A clumped particle model for rock," International Journal of Rock Mechanics and Mining Sciences, vol. 44, no. 7, pp. 997-1010, 2007.

[18] Z. Meng, Numerical Simulation Research on Damage of Rock Strength Caused by Microwave Irradiation, Xi'an University of Science and Technology, Xi'an, China, 2014 (Chinese).

[19] A. Y. Ali and S. M. Bradshaw, "Effect of grain size and thermomechanical properties of minerals on strength reduction of binary ores subjected to microwave irradiation," in Proceedings of the 1st Southern Hemisphere International Rock mechanics Symposium, vol. 2, pp. 85-97, Perth, Australia, 2008.

[20] D. A. Jones, S. W. Kingman, D. N. Whittles, and I. S. Lowndes, "Understanding microwave assisted breakage," Minerals Engineering, vol. 18, no. 7, pp. 659-669, 2005. 

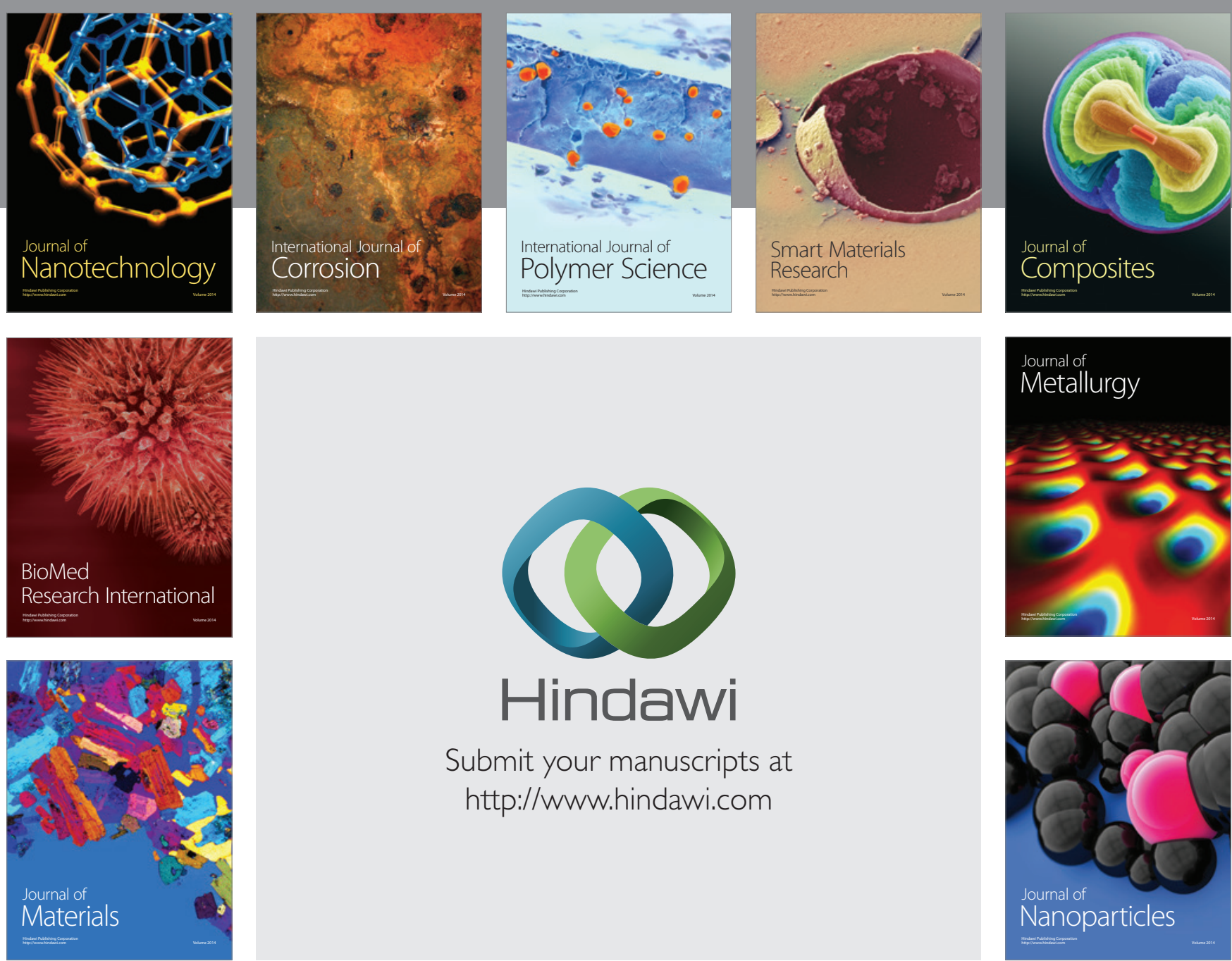

\section{Hindawi}

Submit your manuscripts at

http://www.hindawi.com

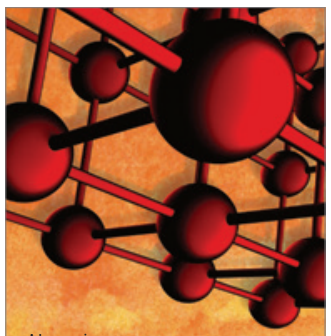

Materials Science and Engineering
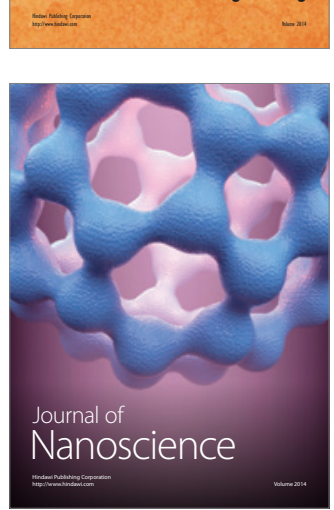
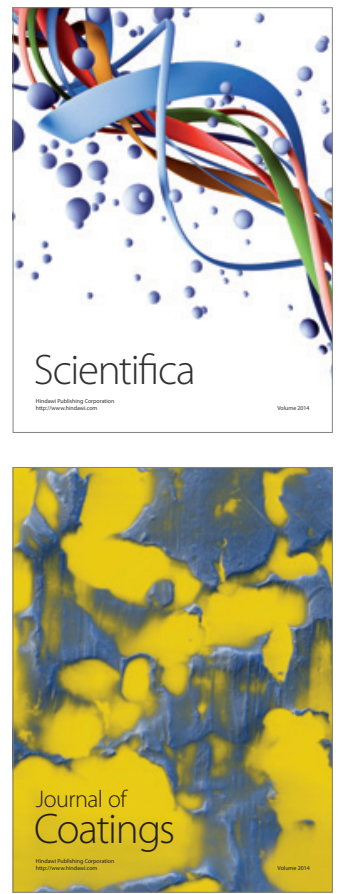
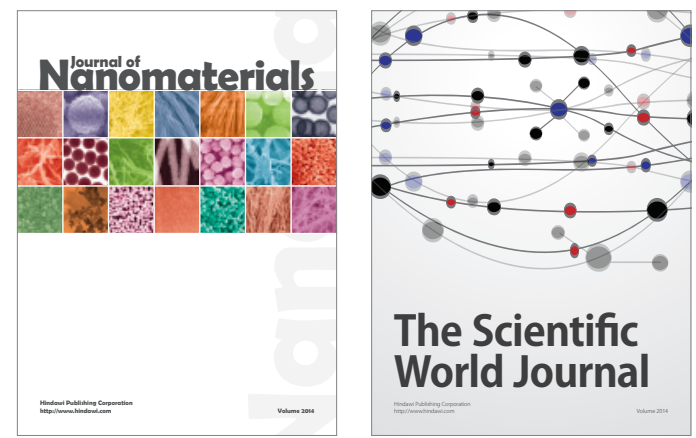

The Scientific World Journal
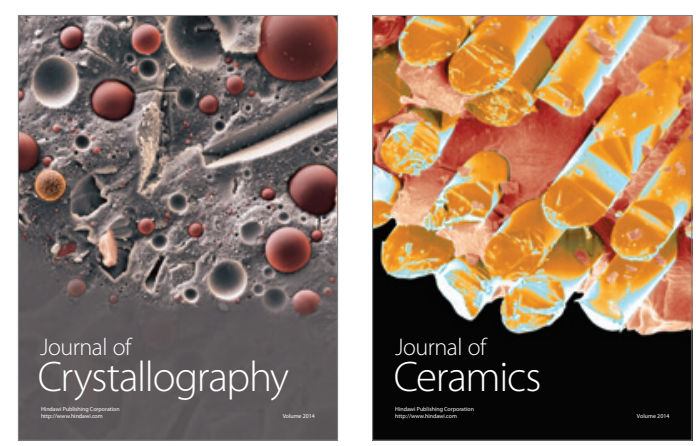
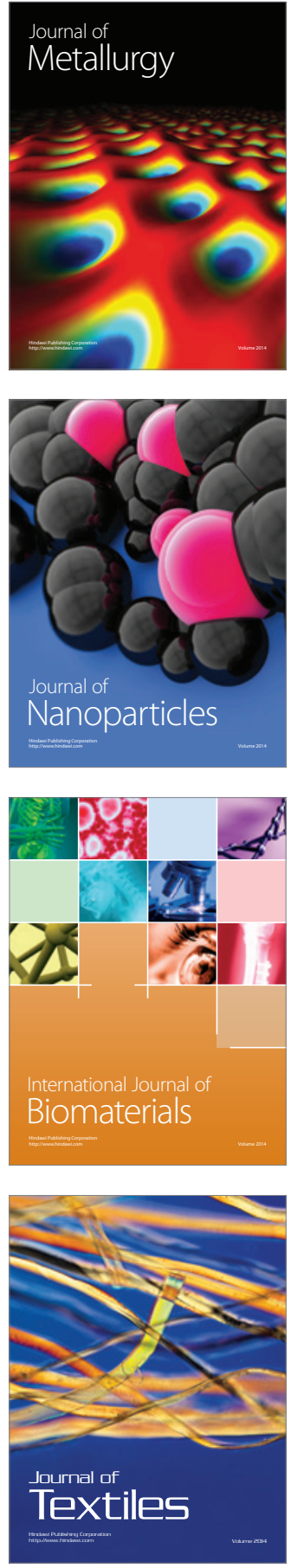